\title{
A Non-linear Generalisation of PDMs using Polynomial Regression
}

\author{
P.D. Sozou, T.F. Cootes, C.J. Taylor and E.C. Di-Mauro \\ Wolfson Image Analysis Unit, Department of Medical Biophysics \\ University of Manchester, M13 9PT, U.K.
}

\begin{abstract}
We have previously described how to model shape variability by means of point distribution models (PDMs) in which there is a linear relationship between a set of shape parameters and the positions of points on the shape. This linear formulation can fail for shapes which articulate or bend: we show examples of such failure for both real and synthetic classes of shape. A new, more general formulation for PDMs, based on polynomial regression, is presented. The resulting Polynomial Regression PDMs (PRPDMs) perform well on the data for which the linear method failed.
\end{abstract}

\section{Introduction}

Deformable template models have proved an effective basis for interpreting images of objects whose appearance can vary. Various approaches have been described [1-6] with varying degrees of generality. One of the most important issues is that of limiting shape variability to that which is consistent with the class of objects to be modelled. $[2,4,7]$.

We have previously described how the shape variability for a class of objects can be represented by Point Distribution Models (PDMs) [8]. The objects are defined by landmark points which are placed in the same way on each of a set of examples. A statistical analysis yields estimates for the mean shape and the main 'modes of variation' for the class. Each mode changes the shape by moving the landmarks along straight lines passing through their mean positions. New shapes, consistent with those found in the training set, are created by modifying the mean shape with weighted sums of the modes.

These linear models have proved successful in capturing the variability present in both natural and man-made objects [7-10]; there are, however, situations where they fail. For example, flexible or articulated objects which can bend through large angles are poorly modelled. The result of such failure is lack of specificity - the resulting models can adopt implausible shapes, very different from those in the training set.

In this paper we describe a method for extending PDMs to deal with non-linear shape variability, by finding modes of variation in which the landmark points move along polynomial paths. We first illustrate how linear PDMs can fail, using examples based on both synthetic and real data. We describe the formulation of Polynomial Regression PDMs (PRPDMs) and show how they can be trained from sets of examples. We also show how a new example can be reconstructed given a set of shape parameters. The method is applied to the two datasets for which the linear method was unsuccessful and is shown to perform well.

\section{Linear Point Distribution Models}

A shape may be conveniently represented by the positions $\mathbf{x}$ of a set of $n$ landmark points; $\mathbf{x}=\left(x_{1}, y_{1} \ldots . x_{n}, y_{n}\right)$. Assume that we have a set of $N$ examples from a given 
class of shape. We place landmarks at corresponding positions on each example $i$ [11] $\left(\mathbf{x}_{i}=\left(x_{i .1}, y_{i .1}, \ldots, x_{i . n}, y_{i . n}\right)^{\mathrm{T}}\right)$. A linear PDM is constructed as follows. The shapes are aligned by scaling, translating and rotating each example so that they overlap as much as possible (see[8]). Let $\overline{\mathbf{x}}$ be the mean of the $N$ aligned examples. Let $\mathbf{S}$ be the covariance matrix of the set of examples about the mean:

$$
\mathbf{S}=\frac{1}{N} \sum_{i=1}^{N}\left(\mathbf{x}_{i}-\overline{\mathbf{x}}\right)\left(\mathbf{x}_{i}-\overrightarrow{\mathbf{x}}\right)^{\mathrm{T}}
$$

The $t$ eigenvectors of $\mathbf{S}$ corresponding to the largest $t$ eigenvalues give a set of basis vectors for a flexible model. A new example is generated by adding to the mean shape a superposition of these basis vectors, weighted by a set of $t$ shape parameters $\left(b_{1}, b_{2}, \ldots b_{t}\right)$. We describe the basis vectors as the modes of variation of the shape.

\section{Failure of the Linear Model}

In many cases a linear PDM can provide a good model of a class of shapes $[9,10]$. This will not, however, always be the case. An obvious example is where one subpart of a modelled object can rotate about another. The linear paths traced out by the landmark points, as each parameter $b_{j}$ is varied, provide a poor approximation to the circular arcs which are required to model the shape variation accurately. The problem manifests itself as a dependency between the shape parameters $b_{j}$. The modes of variation are of course guaranteed to be linearly independent, implying that a 'legal' shape will result when the parameters are chosen independently. If, however, there are non-linear dependencies between the landmark ordinates $\mathbf{x}$, the shape parameters can no longer be chosen independently. We illustrate this problem with two examples.

\subsection{Tadpoles}

The tadpoles shown in Fig. 1 are examples from a synthetic class of shapes. Aflexible spine of 10 segments is generated, with the angle between successive segments varying by a random amount between $-0.12 \mathrm{rad}$ and $+0.12 \mathrm{rad}$ about a mean value $\bar{A}$, which is set at random for each tadpole to between $-0.4 \mathrm{rad}$ and $0.4 \mathrm{rad}$. The higher the value of $|\bar{A}|$ for the tadpole, the greater its tendency to curl up systematically in one direction. The head is represented by six landmark points at one end of the spine. The tail is fleshed out with two landmark points either side of the spine nodes, and one landmark point at the tip. The size of the head and the width of the tail are kept constant.
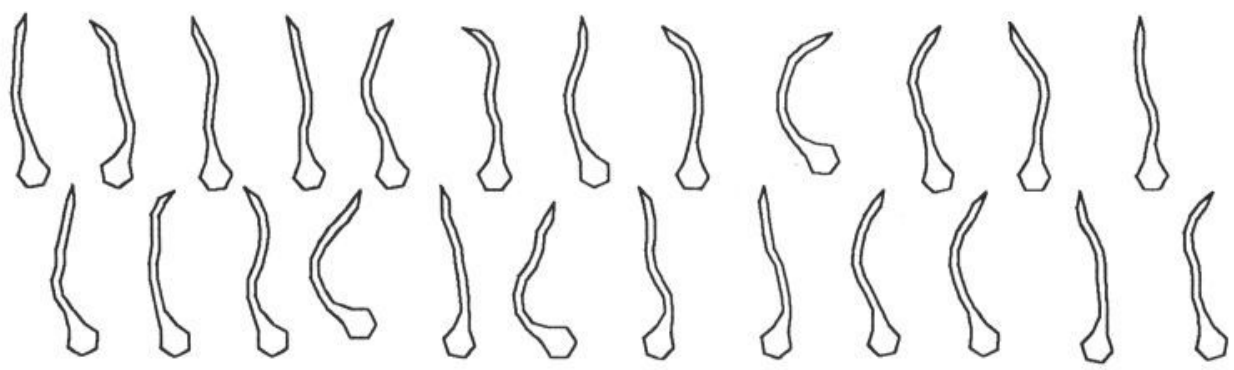

Figure 1. Examples of tadpole training data 
Training a linear PDM on 100 tadpoles, we find that three modes of variation are needed to explain $95 \%$ of the variance in the training data. Fig. 2 demonstrates these modes, showing how the shape changes when each shape parameter is varied by two standard deviations either side of the mean shape. The first mode primarily describes bending, but this is accompanied by lengthening of the tail and an increase in the size of the head. These effects do not occur in the training examples; they are a consequence of using a linear model, in which the landmark points must move in straight lines for each mode of variation. The third mode 'compensates' for the unwanted variation introduced by the first. An alternative way of visualising this problem is provided by Fig. 3 , which shows scattergrams of the second and third shape parameters plotted against the first. A weak non-linear relationship exists between $b_{2}$ and $b_{1}$, with $b_{2}$ tending to be higher when $b_{1}$ is close to zero; there is a very clear non-linear relationship between $b_{3}$ and $b_{1}$, with $b_{3}$ tending to be higher when $b_{1}$ is close to zero. Thus the assumption of independence of the shape parameters does not hold. This means that a combination of shape parameters can be chosen, each within its normal range, such that a shape very different from any in the training set is generated.

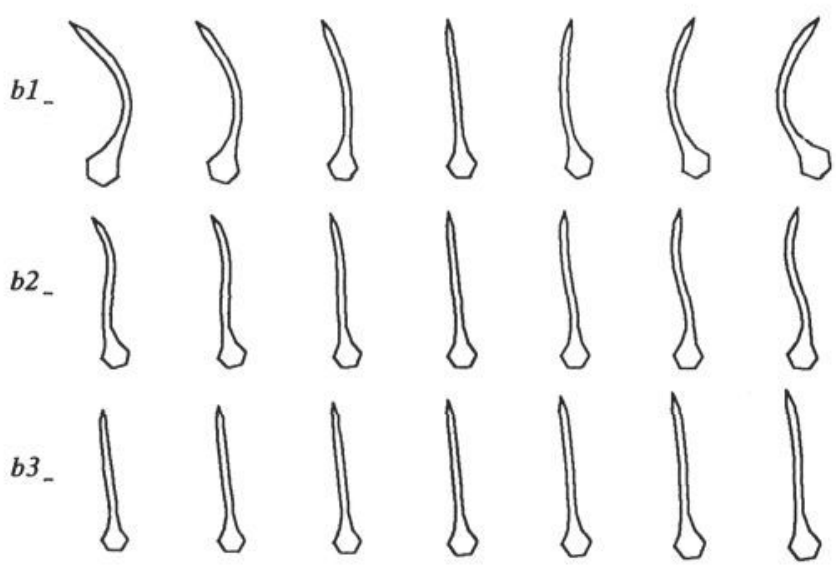

Figure 2. Three modes of variation (mean +-2 standard deviations) of the Linear PDM, trained on the tadpole data. Note the changing size of the head, and the change in tail length which accompanies bending.

(a)

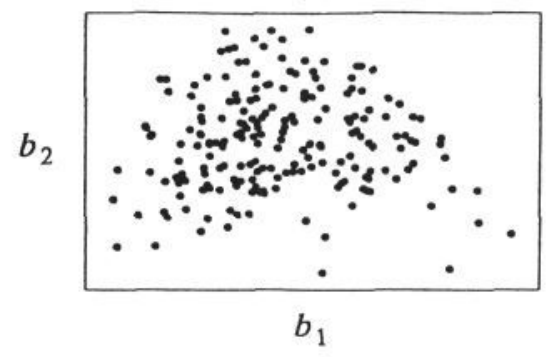

(b)

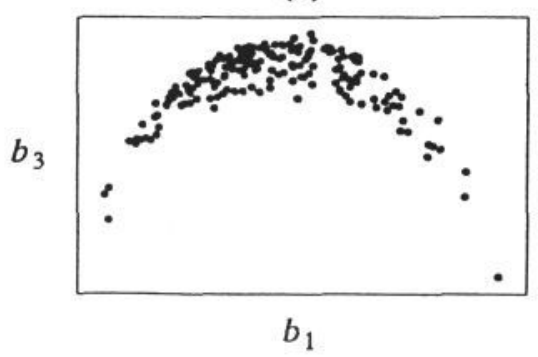

Figure 3. Tadpole data, Linear PDM: scattergrams: (a) $b_{2}$ against $b_{1},(b) b_{3}$ against $b_{1}$. 


\subsection{Chromosomes}

The chromosome data were obtained from microscope images of stained human chromosomes in the late prophase stage of mitosis. Each chromosome is represented by 92 landmark points, placed at opposite ends of 45 equally spaced chords and at the two end points. The procedure for marking the landmark points has been described by Charters [11]. Fig. 4 shows examples from the training set. The up-down and left-right orientation of each chromosome is arbitrary; for this reason we include in the training set a $180^{\circ}$ rotation and two reflections of each image, as well as the original. From 353 distinct images, we thus have 1412 training examples.

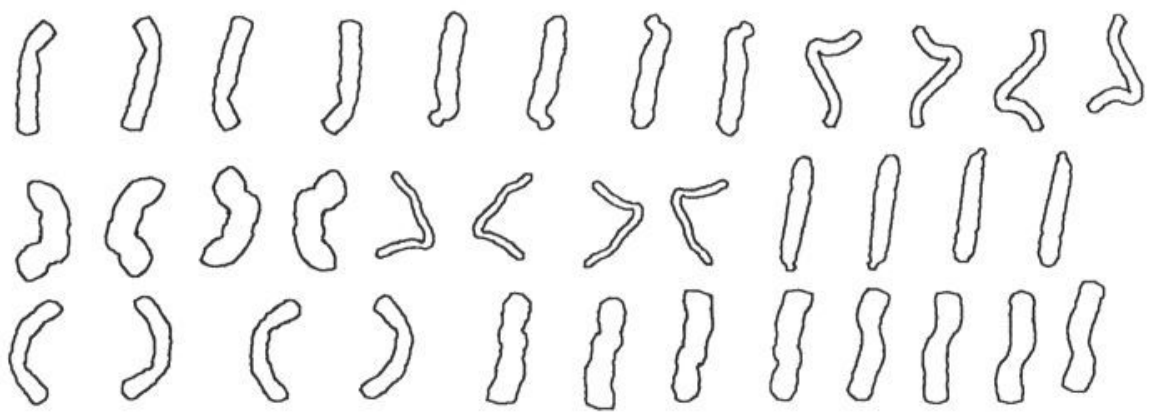

Figure 4. Examples of chromosome training data. Each distinct image appears four times in the training set, owing to reflections and rotation.

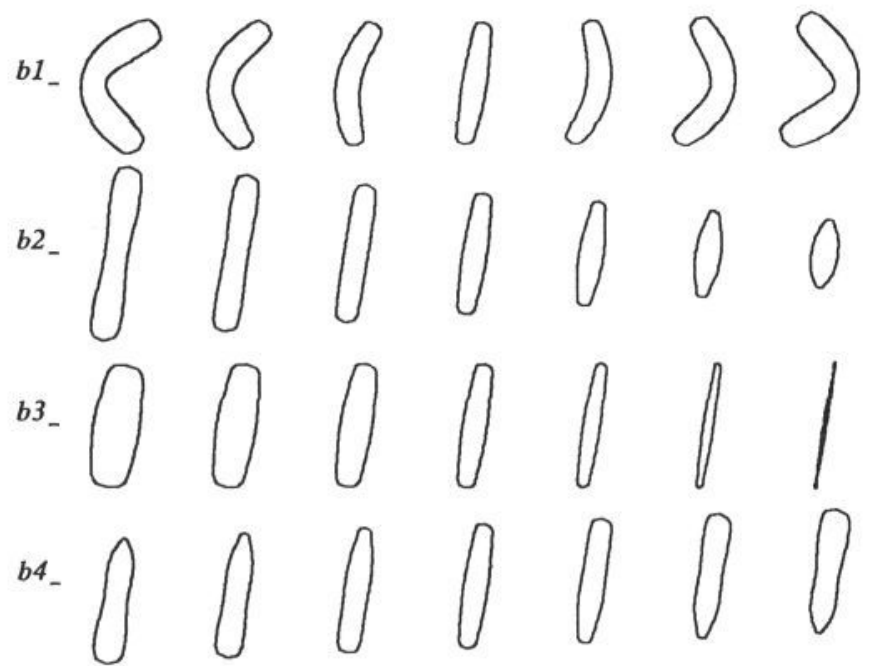

Figl:e 5. First four modes (mean +-2 standard deviations) of linear PDM, trained on the chromosome data. Note the change in length which accompanies bending..

For the linear PDM, eight modes of variation are needed to explain $95 \%$ of the variance in the data. The first four modes are illustrated in Figure 5. We see that, as with the linear tadpole PDM, the bending of the first mode is accompanied by an 
increase in length, whereas the length does not change in this way for the scaled training examples. Fig. 6 is a scattergram of the first two shape parameters $\left(b_{2}\right.$ against $b_{1}$ ) for the training data. There is a very clear non-linear relationship between $b_{2}$ and $b_{1}$, with $b_{2}$ tending to be lower when $b_{1}$ is close to zero: thus the shape parameters are not independent. Again, implausible shapes can be generated by choosing apparently legal combinations of parameters.

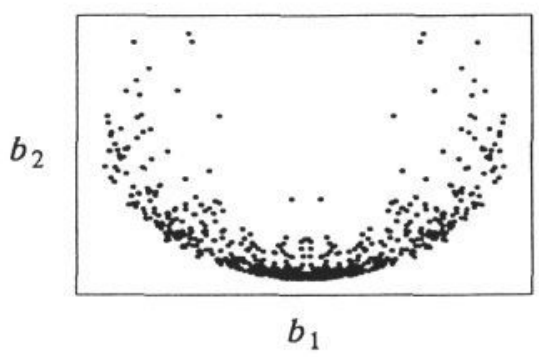

Figure 6. Chromosome data, linear PDM: scattergram of $b_{2}$ against $b_{1}$.

\section{Polynomial Regression Models}

We propose a modified class of models which we call Polynomial Regression PDMs (PRPDMs). The basic idea is to allow landmark points to follow polynomial rather than linear paths as each shape parameter is varied, allowing more complex behaviour, such as bending, to be modelled directly. Our approach is motivated by noting that the eigen-analysis used to extract the modes of variation for a standard PDM can be conceptualised (however implemented) as a sequential process:

(i) For each training example $i$ initialise a vector of residuals $\mathbf{r}_{i}$ to the deviation of that example from the mean: $\mathbf{r}_{i}=\mathbf{x}_{i}-\overline{\mathbf{x}}$.

(ii) Fit a straight line $\mathbf{u}$ to the set of residuals $\left\{\mathbf{r}_{1} \ldots \mathbf{r}_{N}\right\}$, so as to minimise the sum of squares of distances from the straight line.

(iii) Compute the residual deviation from the line $\mathbf{u}$ (i.e remove the component of the $\mathbf{r}_{i}$ along $\mathbf{u}$ ) to give a new set of residuals.

$$
\mathbf{r}_{i}^{\text {new }}=\mathbf{r}_{i}-\left(\mathbf{r}_{i} \cdot \mathbf{u}\right) \mathbf{u}
$$

Steps (ii) and (iii) are repeated to find subsequent modes. We normally fit a sufficient number of modes so that the remaining residuals are small enough to be attributed to random noise - typically when the unexplained variance is between $5 \%$ and $1 \%$ of the original variance.

We note that, once a linear mode has been extracted, it may be possible to further reduce the residuals $\mathbf{r}_{i}$ without introducing additional modes of variation by fitting a polynomial along the direction of the mode. For example in Fig. 3, $b_{2}$ and $b_{3}$ could be modelled by polynomials in $b_{1}$. This leads to the idea of a polynomial regression model. The details of the procedure for building a PRPDM are as follows: 
(i) Compute the initial variance of the data, $\sigma_{1}^{2}$. Then, for each training example, initialise a vector of residuals $\mathbf{r}_{i}$ to the vector of landmark positions $\mathbf{x}_{i}$. (i.e. let $\mathbf{r}_{i}$ $=\mathbf{x}_{i}$.)

(ii) Let the current residual vector for each example $i$ be denoted by $\mathbf{r}_{i}$, let the mean value of the $\mathbf{r}_{i}$ be denoted by $\overline{\mathbf{r}}$, and let the covariance matrix of the set of current residual vectors $\left\{\mathbf{r}_{1}, \mathbf{r}_{2}, \ldots \mathbf{r}_{N}\right\}$ about the mean be denoted by $\mathbf{S}$ :

$$
\mathbf{S}=\frac{1}{N} \sum_{i=1}^{N}\left(\mathbf{r}_{i}-\overrightarrow{\mathbf{r}}\right)\left(\mathbf{r}_{i}-\overrightarrow{\mathbf{r}}\right)^{\mathrm{T}}
$$

Find the eigenvectors of $\mathbf{S}$, which we denote by $\left\{\mathbf{p}_{1}, \mathbf{p}_{2} \ldots\right\}$. Define $d$, the true linear dimensionality of the set of residuals, as the number of eigenvectors needed to explain all of the variance in the residuals, apart from a tiny amount (which we set to $10^{-10} \sigma_{\mathrm{I}}^{2}$ ) which may be considered as arising from rounding errors.

(iii) Transform the $\mathbf{r}_{i}$ to a coordinate system with the axes lying along the eigenvectors, with $d$ ordinates (corresponding to the $d$ eigenvectors which explain all the variance other than that due to rounding errors). The new coordinates can then be labelled as

$$
\mathbf{e}_{i}=\left(e_{i, 1}, e_{i, 2} \ldots e_{i, d}\right)=\mathbf{P}^{\mathrm{T}}\left(\mathbf{r}_{i}-\overrightarrow{\mathbf{r}}\right)
$$

where $\mathbf{P}=\left(\mathbf{p}_{1}\left|\mathbf{p}_{2}\right| \ldots \mid \mathbf{p}_{d}\right)$. If the $k$ th mode is being fitted, the first $e$-value $e_{i, 1}$ is the $k$ th shape parameter of this model for example $i$.

(iv) Attempt to fit non-linear functions to the data, modelling the second and subsequent $\boldsymbol{e}$-values as non-linear functions of the first.

$$
e_{i, j}^{*}=f_{j}\left(e_{i, 1}\right) \quad \text { where } 2 \leq j \leq d
$$

The nonlinear functions used are polynomials:

$$
f_{j}\left(e_{i, 1}\right)=a_{0, j}+\left(a_{1, j}\right)\left(e_{i, 1}\right)+\left(a_{2, j}\right)\left(e_{i, 1}\right)^{2} \ldots+\left(a_{m, j}\right)\left(e_{i, 1}\right)^{n}
$$

where $m$ is the order of the fitted polynomials for the mode in question. The coefficients $a_{0 j}, a_{1 j}$ etc are chosen so as to minimise the residual error $E$ given by:

where

$$
E=\sum_{j=2}^{d} E_{j}
$$

$$
E_{j}=\sum_{i=1}^{N}\left(e_{i, j}^{*}-e_{i, j}\right)^{2}
$$

The coefficients $a_{t j}$ (where $0 \leq t \leq m$, and $2 \leq j \leq d$ ) are obtained by partially differentiating $E_{j}$ with respect to each $a_{t j}$, and setting the derivative 
equal to zero: this gives $m+1$ linear equations in $m+1$ unknowns, which can be solved by standard methods.

(v) Store the mean residual $\overline{\mathbf{r}}$, the eigenvectors $\left\{\mathbf{p}_{1}, \mathbf{p}_{2}, \ldots \mathbf{p}_{d}\right\}$ and the coefficients $a_{t j}$.

(vi) Compute the new residual vectors $\mathbf{r}_{i}^{\text {new }}$ whose components are given by the difference between the data $e$-value $e_{i, j}$ and the modelled value $e_{i j}^{*}$. Note that by definition the first $e$-value $e_{i, 1}$ is the same for the model and the data. We therefore ignore this zero component and set the first component of the new residual vector as the residual error in the second $e$-value. The other components are similarly shifted down, and thus the new residuals will have $d-1$ components, given by

$$
r_{i j}^{\text {new }}=e_{i j+1}-e_{i j+1}^{\cdot}
$$

where $1 \leq j \leq d-1$

Steps (ii) to (vi) are repeated to fit subsequent modes, until the residual errors are small enough to be attributed to random noise - as with the linear model this is typically when the unexplained variance is between $5 \%$ and $1 \%$ of the original variance $\sigma_{\mathrm{I}}^{2}$.

To compute the shape parameters (" $b$-values") for a new shape (i.e. a new set of landmark point positions), we repeat the above steps, going through the modes sequentially, but we use the eigenvectors $\mathbf{p}$ and the polynomial coefficients $a$ that were stored during training for the coordinate transformations.

To compute a shape instance (i.e a set of landmark point coordinates) from a set of shape parameters, we reverse the above procedure. We evaluate the modes sequentially, beginning with the last mode to be fitted (given by the last shape parameter) first, and the first mode to be fitted (first shape parameter) last, again using the stored eigenvectors $\mathbf{p}$ and polynomial coefficients $a$ for each mode. The steps for evaluating each mode are as follows:

(i) Read in the mean residual $\overline{\mathbf{r}}$, the true linear dimensionality $d$, the eigenvectors $\left\{\mathbf{p}_{1}, \ldots \mathbf{p}_{d}\right\}$, the degree of polynomial $m$ and the polynomial coefficients $a_{t j}$ corresponding to the mode to be fitted.

(ii) Set up a new vector e (of $d$ components) of $e$-values. The first component is set to the shape parameter $b$ corresponding to the mode being evaluated. (For the first mode to be evaluated, this will be the last shape parameter. For the last mode to be evaluated, it will be the first shape parameter.) The remaining components are determined by reversing equation (2), so that they are given by the polynomial function of $e_{1}$, to which (if applicable, i.e. if other modes have been evaluated) is added the appropriate component of the "residual" $\mathbf{r}$ :

$$
e_{j}=a_{0 j}+\left(a_{1 j}\right)\left(e_{1}\right) \ldots+a_{m j}\left(e_{1}\right)^{m}\left[+r_{i j-1}\right]
$$

(iii) Compute a new "residual" vector $\mathbf{r}$, given by a superposition of the the eigenvectors $\left\{\mathbf{p}_{1}, \ldots \mathbf{p}_{d}\right\}$, weighted according to the $e$-values $\left\{e_{1}, \ldots e_{d}\right\}$, 
added to the mean $\overline{\mathbf{r}}$ which was stored during training. (This is a reversal of the eigenvalue decomposition used in building the PRPDM.):

$$
\mathbf{r}^{\text {new }}=\overline{\mathbf{r}}+\mathbf{p}_{1} e_{1}+\mathbf{p}_{2} e_{2} \ldots+\mathbf{p}_{d} e_{d}=\overline{\mathbf{r}}+\mathbf{P e}
$$

Steps (i) to (iii) are repeated until we have evaluated the mode corresponding to the first shape parameter. The landmark position vector is then simply given by the final $\mathbf{r}$.

\section{Results}

\subsection{Tadpoles}

We have trained a PRPDM on the tadpole data, using polynomials of degree 2 (i.e quadratics) for each mode. We find that two modes of variation are sufficient to describe $95 \%$ of the variance in the data. The modes are shown in Fig. 7 . The first mode gives a sensible representation of bending, with the tip of the tail following a curved path, and there is less spurious variation in the size of the head. The second mode of the PRPDM is similar to the second mode of the Linear PDM. No 'compensation' mode is required for the PRPDM. A scattergram of the two shape parameters of the PRPDM is shown in Fig.8: the shape parameters appear to be more independent than for the linear PDM.
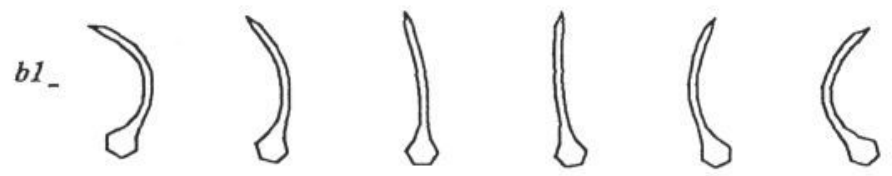

b2.
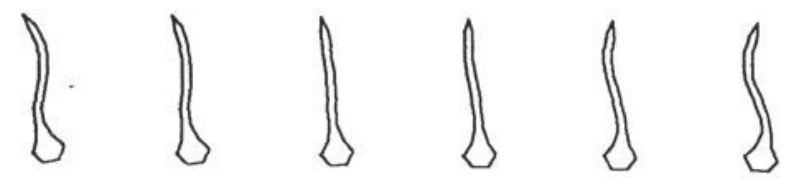

Figure 7. Two modes (mean +-2 standard deviations) of polynomial regression PDM, trained on the tadpole data. The bending is more accurately captured by this model than by the linear PDM, and there is less spurious variation in the size of the head.

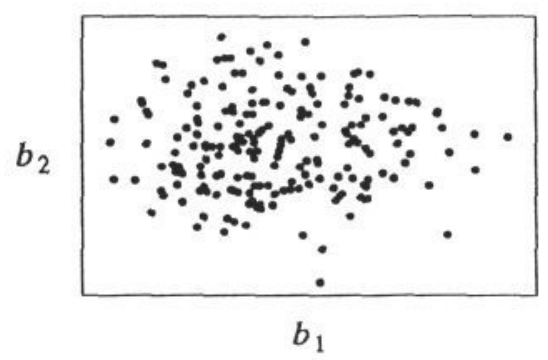

Figure 8. Tadpole data, PRPDM: scattergram of $b_{2}$ against $b_{1}$. 


\subsection{Chromosomes}

We have trained a PRPDM on the chromosome data using polynomials of degree 2 for each mode. We find that, as for the linear PDM, eight modes of variation are needed to explain $95 \%$ of the variance in the data. The first four modes are shown in Fig. 9. As with the tadpole data we see that the first mode of the polynomial regression PDM gives a sensible representation of bending. This improvement in the model is borne out in the scattergram of the first two shape parameters (Fig. 10), which are clearly more independent than the first two parameters of the linear PDM (Fig. 6). The other modes of Fig. 9 are qualitatively similar to those of the linear model.

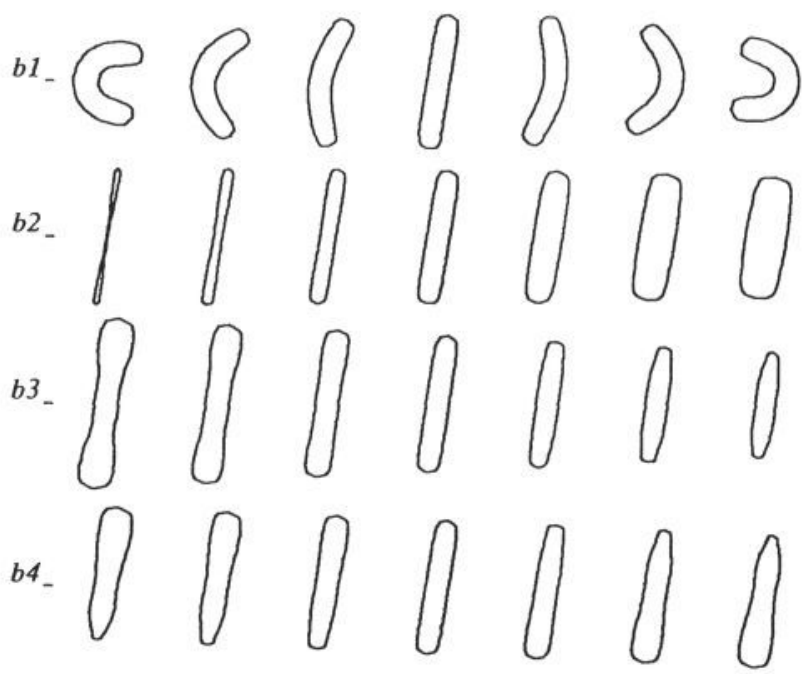

Figure 9. First four modes (mean +-2 s.d..) of PRPDM, trained on the chromosome data. The first (bending) mode is more accurately captured by this model than by the linear PDM.

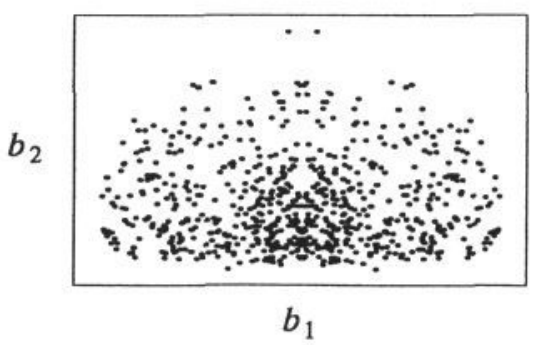

Figure 10. Chromosome data, PRPDM: scattergram of $b_{2}$ against $b_{1}$.

\section{Discussion}

We have formulated and tested PRPDMs - shape models which are more general than the linear PDMs which we have described previously. PRPDMs can generate 
non-linear modes of shape variation by modelling the loci of landmark points using polynomial functions. We have shown that for two classes of shape, one synthetic and one based on real image data, a PRPDM gives a more accurate representation of the shape variation than the linear PDM, leading to a more specific model. A PRPDM can be used directly for image interpretation in Active Shape Model search [7].

Apart from examples such as those considered above, an obvious application for these non-linear models is in the interpretation of 2D images of variable 3D objects. We can already model small changes in viewpoint using linear PDMs; we anticipate that larger changes in viewpoint will be satisfactorily modelled using a PRPDM. We note however that the PRPDM will not necessarily succeed for all classes of non-linear shape - it would for example fail if the training examples occupied the surface of an ellipsoid in "shape-space". We are currently investigating other forms of non-linear shape model.

\section{Acknowledgments}

PDS, TFC and ECD were supported by the Science and Engineering Research Council. We thank Graham Charters for providing the chromosome data.

\section{References}

1. M. Kass, A. Witkin and D. Terzopoulos, "Snakes: active contour models", Proc. First International Conference on Computer Vision 259-268 (IEEE Computer Society Press, 1987).

2. L.H. Staib and J.S.Duncan, "Parametrically deformable contour models", IEEE Computer Society Conference on Computer Vision and Pattern Recognition, San Diego, USA 427-430 (1989).

3. P. Lipson, A.L Yuille, D. O'Keefe, J. Cavanaugh, J. Taafe, and D. Rosenthal, "Deformable templates for feature extraction from medical images", Proc. First European Conference on Computer Vision (Lecture Notes in Computer Science, O. Faugeras Ed.) 413-417 (Springer-Verlag, 1990).

4. U. Grenandier, Y. Chow and D.M.Keenan, Hands. A Patterm-Theoretic Study of Biological Shapes (SpringerVerlag, New York, 1991).

5. A. Pentland and S. Schlaroff, "Closed-form solutions for physically-based modeling and recognition", IEEE Trans. Pattern Anal. Mach. Intell. 13, 715-729 (1991).

6. D. Terzopoulos and D. Metaxas, "Dynamic 3D models with local and global deformations: deformable superquadrics”, IEEE Trans. Pattern Anal. Mach. Intell. 13, 703-714 (1991).

7. T.F. Cootes, C.J. Taylor, D.H. Cooper and J. Graham, "Active shape models - their training and application", CVGIP, Image Understanding (in press).

8. T.F. Cootes, C.J. Taylor, D.H. Cooper and J. Graham, "Training models of shape from sets of examples”, Proc. BMVC92, Springer-Verlag 9-18 (1992).

9. A. Hill, Model-based image interpretation using genetic algorithms", Image and Vision Computing 10, 295-300 (1992).

10. A. Lanitis, C.J. Taylor and T.F. Cootes, "A generic system for classifying variable objects using flexible template matching”, Proc. BMVC92, BMVA Press 329-338 (1993).

11. G.C. Charters, "Combination of evidence in the analysis of prophase chromosomes using computer vision", Wolfson Image Analysis Unit, University of Manchester, internal report (September 1993). 